\title{
The calculation problem of thermodynamic processes in a steam turbine
}

\author{
Alexander Chistyakov ${ }^{1, *}$, Alla Nikitina $^{2}$ and Elena Protsenko ${ }^{3}$ \\ ${ }^{1}$ Don State Technical University, Rostov, Russia \\ ${ }^{2}$ Southern Federal University, Taganrog, Russia \\ ${ }^{3}$ Taganrog Institute of A.P. Chekhov (branch) of the Rostov State University of Economics, Taganrog, Russia
}

\begin{abstract}
This paper covers the calculation of aerodynamic processes in a steam turbine using the modern information technologies and computational methods, that are contributed to the increasing the accuracy of the calculations. The practical significance is the development and application of the model of aerodynamic processes in a steam turbine, and determination of limits and prospects with using of proposed model. The aerodynamic processes in a turbine are characterized by the air and heat flow non-uniformity, significantly affect the reliability and efficiency of the turbine. The calculation was performed taking into account the complex geometry of the turbine, and can be applied to any turbine of similar design with minor changes.
\end{abstract}

\section{Introduction}

At present, the question about the optimization problem of installation and exploitation of steam turbines is the actual. With the development of modern technology and industry needs to the exploitation of turbines, requirements are the more stringent associated with the reliability and efficiency of their operation. A large number of existing turbines is practically close to the elaboration its resource. Therefore, the introduction of more modern units is required. The fundamentals of the theory of heat transfer and analysis results of transfer processes are required to assess the reliability and efficiency of the facility. Therefore, these data should be taken into account in designing of steam turbines. Thermal systems modelling include the problems of optimal control of thermal modes, due to which we can choose the best from different implementations. The optimization of thermal modes is reduced to the solution of heat conduction problem. Mathematical modelling of thermal processes in technogenic systems is relevant at the present. Due to it, we can check the correctness of engineering ideas and correct errors at the stage of designing by the simple, low-cost means. The mathematical model is represented by the scheme «model - algorithm - program», and must contain the structure, characteristic features of the process and should be described by the equation system or functional relations. After the designing, it is necessary to determine the real values of temperature at significant points of the steam facility and analyze the compliance with required values.

\section{Problem statement}

The main equations of air dynamics are: - Navier-Stokes motion equations

$$
\begin{gathered}
\frac{\partial v_{x}}{\partial t}+v_{x} \frac{\partial v_{x}}{\partial x}+v_{y} \frac{\partial v_{x}}{\partial y}+v_{z} \frac{\partial v_{x}}{\partial z}= \\
-\frac{1}{\rho} \frac{\partial P}{\partial x}+\mu\left(\frac{\partial^{2} v_{x}}{\partial x^{2}}+\frac{\partial^{2} v_{x}}{\partial y^{2}}+\frac{\partial^{2} v_{x}}{\partial z^{2}}\right)+f_{x}, \\
\frac{\partial v_{y}}{\partial t}+v_{x} \frac{\partial v_{y}}{\partial x}+v_{y} \frac{\partial v_{y}}{\partial y}+v_{z} \frac{\partial v_{y}}{\partial z}= \\
=-\frac{1}{\rho} \frac{\partial P}{\partial y}+\mu\left(\frac{\partial^{2} v_{y}}{\partial x^{2}}+\frac{\partial^{2} v_{y}}{\partial y^{2}}+\frac{\partial^{2} v_{y}}{\partial z^{2}}\right)+f_{y}, \\
\frac{\partial v_{z}}{\partial t}+v_{x} \frac{\partial v_{z}}{\partial x}+v_{y} \frac{\partial v_{z}}{\partial y}+v_{z} \frac{\partial v_{z}}{\partial z}= \\
=-\frac{1}{\rho} \frac{\partial P}{\partial z}+\mu\left(\frac{\partial^{2} v_{z}}{\partial x^{2}}+\frac{\partial^{2} v_{z}}{\partial y^{2}}+\frac{\partial^{2} v_{z}}{\partial z^{2}}\right)+f_{z},
\end{gathered}
$$

- continuity equation

$$
\rho_{t}^{\prime}+(\rho u)_{x}^{\prime}+(\rho v)_{y}^{\prime}+(\rho w)_{z}^{\prime}=0 .
$$

- state equation

$$
P=\varepsilon \frac{\rho}{M} R T,
$$

where $\mathbf{V}=\left\{v_{x}, v_{y}, v_{z}\right\}$ are velocity vector components; $p$ is a pressure; $\rho$ is a density; $M$ is the molar mass; $R$ is the universal gas constant; $\mu$ is the turbulent exchange coefficient; $T$ is the temperature, $\varepsilon$ is the coefficient describing the deviation of pressure superheated steam from the ideal gas.

\footnotetext{
* Corresponding author: cheese_05@mail.ru
} 
We assume that the air environment is initially in quiescence. Therefore, the initial conditions have the form:

$$
u=0, w=0, P=P_{a},
$$

where $P_{a}$ is the initial pressure.

The system of equations (1) and (2) are considered with the following boundary conditions:

- on an impervious boundary

$$
\begin{gathered}
\rho_{w} \eta \frac{\partial v_{x}}{\partial n}=\tau_{x, b}(t), \rho_{w} \eta \frac{\partial v_{y}}{\partial n}=\tau_{y, b}(t), \rho_{w} \eta \frac{\partial v_{z}}{\partial n}=\tau_{z, b}(t), \\
\mathbf{V}_{n}=0, P_{n}^{\prime}=0 ;
\end{gathered}
$$

- on the lateral permeable borders

$$
\frac{\partial v_{x}}{\partial n}=0, \frac{\partial v_{y}}{\partial n}=0, \frac{\partial v_{z}}{\partial n}=0, \frac{\partial P}{\partial n}=0 ;
$$

- on the source

$$
v_{x}=U, v_{y}=V, v_{z}=W, \frac{\partial P}{\partial n}=0 ;
$$

where $U, V, W$ are components of velocity vector on the source; $\tau_{x}, \tau_{z}$ are components of tangential shear stress.

The system of Navier-Stokes' equations in the cylindrical coordinate system has the form:

$$
\begin{gathered}
\frac{\partial v_{r}}{\partial t}+v_{r} \frac{\partial v_{r}}{\partial r}+\frac{v_{\theta}}{r} \frac{\partial v_{r}}{\partial \theta}-\frac{v_{\theta}^{2}}{r}+v_{z} \frac{\partial v_{r}}{\partial z}=-\frac{1}{\rho} \frac{\partial P}{\partial r}+ \\
+\mu\left(\frac{\partial}{\partial r}\left(\frac{1}{r} \frac{\partial\left(r v_{r}\right)}{\partial r}\right)+\frac{1}{r^{2}} \frac{\partial^{2} v_{r}}{\partial \theta^{2}}-\frac{2}{r^{2}} \frac{\partial v_{\theta}}{\partial \theta}+\frac{\partial^{2} v_{r}}{\partial z^{2}}\right)+f_{r} \\
\frac{\partial v_{\theta}}{\partial t}+v_{r} \frac{\partial v_{\theta}}{\partial r}+\frac{v_{\theta}}{r} \frac{\partial v_{\theta}}{\partial \theta}+\frac{v_{\theta} v_{r}}{r}+v_{z} \frac{\partial v_{\theta}}{\partial z}=-\frac{1}{r \rho} \frac{\partial P}{\partial \theta}+ \\
+\mu\left(\frac{\partial}{\partial r}\left(\frac{1}{r} \frac{\partial\left(r v_{\theta}\right)}{\partial r}\right)+\frac{1}{r^{2}} \frac{\partial^{2} v_{\theta}}{\partial \theta^{2}}+\frac{2}{r^{2}} \frac{\partial v_{r}}{\partial \theta}+\frac{\partial^{2} v_{\theta}}{\partial z^{2}}\right)+f_{\theta}, \\
\quad \frac{\partial v_{z}}{\partial t}+v_{r} \frac{\partial v_{z}}{\partial r}+\frac{v_{\theta}}{r} \frac{\partial v_{z}}{\partial \theta}+v_{z} \frac{\partial v_{z}}{\partial z}=-\frac{1}{\rho} \frac{\partial P}{\partial z}+ \\
+\mu\left(\frac{1}{r} \frac{\partial}{\partial r}\left(r \frac{\partial v_{z}}{\partial r}\right)+\frac{1}{r^{2}} \frac{\partial^{2} v_{z}}{\partial \theta^{2}}+\frac{\partial^{2} v_{z}}{\partial z^{2}}\right)+f_{z}
\end{gathered}
$$

The system of equations (4) in the case of axial symmetry has the form:

$$
\begin{aligned}
& \frac{\partial v_{r}}{\partial t}+v_{r} \frac{\partial v_{r}}{\partial r}-\frac{v_{\theta}^{2}}{r}+v_{z} \frac{\partial v_{r}}{\partial z}=-\frac{1}{\rho} \frac{\partial P}{\partial r}+ \\
& +\mu\left(\frac{\partial}{\partial r}\left(\frac{1}{r} \frac{\partial\left(r v_{r}\right)}{\partial r}\right)+\frac{\partial^{2} v_{r}}{\partial z^{2}}\right)+f_{r}, \\
& \frac{\partial v_{\theta}}{\partial t}+v_{r} \frac{\partial v_{\theta}}{\partial r}+\frac{v_{\theta} v_{r}}{r}+v_{z} \frac{\partial v_{\theta}}{\partial z}= \\
& =\mu\left(\frac{\partial}{\partial r}\left(\frac{1}{r} \frac{\partial\left(r v_{\theta}\right)}{\partial r}\right)+\frac{\partial^{2} v_{\theta}}{\partial z^{2}}\right)+f_{\theta}, \\
& \frac{\partial v_{z}}{\partial t}+v_{r} \frac{\partial v_{z}}{\partial r}+v_{z} \frac{\partial v_{z}}{\partial z}=-\frac{1}{\rho} \frac{\partial P}{\partial z}+ \\
& +\mu\left(\frac{1}{r} \frac{\partial}{\partial r}\left(r \frac{\partial v_{z}}{\partial r}\right)+\frac{\partial^{2} v_{z}}{\partial z^{2}}\right)+f_{z} .
\end{aligned}
$$

The continuity equation (2) in the cylindrical coordinate system has the form:

$$
\frac{\partial \rho}{\partial t}+\frac{1}{r} \frac{\partial\left(\rho r v_{r}\right)}{\partial r}+\frac{1}{r} \frac{\partial\left(\rho v_{\theta}\right)}{\partial \theta}+\frac{\partial\left(\rho v_{z}\right)}{\partial z}=0,
$$

The equation (5) in the case of axial symmetry has the form:

$$
\frac{\partial \rho}{\partial t}+\frac{1}{r} \frac{\partial\left(\rho r v_{r}\right)}{\partial r}+\frac{\partial\left(\rho v_{z}\right)}{\partial z}=0 .
$$

\section{The state equation}

The main equation for the superheated steam is the equation for the specific Gibbs energy, consisting of two parts - relating to ideallocation as $\gamma^{0}$ and describing real component $\gamma^{r}$ :

$$
\begin{gathered}
g(\mathrm{p}, \mathrm{T}) / \mathrm{RT}=\gamma(\pi, \tau)=\gamma^{0}(\pi, \tau)+\gamma^{r}(\pi, \tau) \\
\gamma^{0}=\ln \pi+\sum_{i=1}^{9} n_{i}^{0} \tau^{J_{i}^{0}} \\
\gamma^{r}=\sum_{i=1}^{43} n_{i} \pi^{I_{i}}(\tau-0,5)^{J_{i}}
\end{gathered}
$$

where $\pi=p / p^{*}$ and $\tau=T^{*} / T, \quad p^{*}=1 M P a$, $T^{*}=540 \mathrm{~K}$.

From the relation for the specific volume

$$
\nu P / R T=\pi \gamma_{\pi}, \gamma_{\pi}=\left(\frac{\partial \gamma}{\partial \pi}\right)_{\tau}
$$

follows the next:

$$
P M=\left(1+\sum_{i=1}^{43} n_{i} I_{i}\left(p / p^{*}\right)^{I_{i}}\left(T^{*} / T-0,5\right)^{J_{i}}\right) \rho R T .
$$

\section{Turbulence model}

We used the model of Abramovich-Sekundow, which takes into account very important factors such as the presence of hard walls, the prehistory of the flow, convective and diffusion turbulent pulsation transfer:

$$
\begin{aligned}
& \frac{\partial v_{\text {турб }}}{\partial t}+\sum_{i=1}^{3} v_{i} \frac{\partial v_{\text {турб }}}{\partial x_{i}}=\sum_{i=1}^{3} \frac{\partial}{\partial x_{i}}\left(\left(v_{\text {мол }}+k v_{\text {турб }}\right) \frac{\partial v_{\text {турб }}}{\partial x_{i}}\right)+ \\
& +v_{\text {турб }} f\left(\frac{v_{\text {турб }}}{8 v_{\text {мог }}}\right) D-\gamma S, S=\frac{v_{\text {турб }}\left(v_{\text {мог }}+\beta v_{\text {мурб }}\right)}{L_{\min }^{2}} \text {, } \\
& D=\sqrt{\sum_{i=1}^{3} \sum_{j} \frac{\partial v_{i}}{\partial x_{j}}\left(\frac{\partial v_{i}}{\partial x_{j}}+\frac{\partial v_{j}}{\partial x_{i}}\right)}, \\
& f(z)=0,2 \frac{z^{2}+1,47 z+0,2}{z^{2}-1,47 z+1},
\end{aligned}
$$

where $k=2, \gamma=50, \beta=0,06, L_{\min }$ is the minimum distance to a solid wall; $v_{\text {мол }}$ is the molecular viscosity, $v_{\text {myp }}$ is the turbulent viscosity.

Turbulence model (10) is considered with the boundary condition $\left.\left(v_{\text {myp }}\right)_{n}^{\prime}(t, x, y, z)\right|_{(x, y, z) \in \Gamma}=0$.

Complete model (10) with initial condition: $\left(v_{\text {myp }}\right)(t, x, y, z)=v_{0}$. 
The next equation is used to calculate values of dynamic viscosity $v_{\text {мол }}$ :

$$
v_{\text {мол }}=\bar{v}_{0}(\tau) \cdot \bar{v}_{1}(\tau, \delta) \cdot v^{*},
$$

where $v^{*}=55,071 \cdot 10^{-6} \mathrm{~Pa} \cdot \mathrm{s} ; \tau=T / T^{*} ; T$ is the temperature, $[\mathrm{K}] ; T^{*}=647,226 \mathrm{~K} ; \delta=\rho / \rho^{*} ; \rho$ is a density, $\left[\mathrm{kg} / \mathrm{m}^{3}\right] ; \rho^{*}=317,763 \mathrm{~kg} / \mathrm{m}^{3} ; \bar{v}_{0}=v_{0} / v^{*} ; v_{0}$ is the dynamic viscosity of water vapor in the limit of zero density

$$
\bar{v}_{0}(\tau)=\tau^{0,5}\left(\sum_{i=0}^{3} H_{i} \tau^{-i}\right)^{-1},
$$

where $H_{0}=1, \quad H_{1}=0,978197, \quad H_{2}=0,579829$, $H_{3}=-0,202354$.

$$
\bar{v}_{1}(\tau, \delta)=\exp \left(\delta \sum_{i=0}^{5} \sum_{j=0}^{6} H_{i, j}(1 / \tau-1)^{i}(\delta-1)^{j}\right) .
$$

\section{The heat conductivity problem}

Thermal processes in turbine $\bar{G}$ are described by the heat conduction equation:

$$
\begin{aligned}
& c \rho \frac{\partial T}{\partial t}+v_{x} \frac{\partial T}{\partial x}+v_{y} \frac{\partial T}{\partial y}+v_{z} \frac{\partial T}{\partial z}=\frac{\partial}{\partial x}\left(\lambda \frac{\partial T}{\partial x}\right)+ \\
& +\frac{\partial}{\partial y}\left(\lambda \frac{\partial T}{\partial y}\right)+\frac{\partial}{\partial z}\left(\lambda \frac{\partial T}{\partial z}\right)+q_{v}(P),
\end{aligned}
$$

which can be written in the case of axial symmetry as: $\rho c r T_{t}^{\prime}+r v_{r} T_{r}^{\prime}+r v_{z} T_{z}^{\prime}=r\left(\lambda T_{z}^{\prime}\right)_{z}^{\prime}+\left(\lambda r T_{r}^{\prime}\right)_{r}^{\prime}+r q_{v}(P)$.

In the system (14), (15): $T$ is the temperature, $[K]$; $\lambda$ is the coefficient of conductivity of water; $\rho$ is the density of the metal; $c$ is the coefficient of the metal conductivity; $r$ is the polar radius; $q_{v}(P)$ is the source function. We will consider equation (15) with boundary conditions of third order:

$$
T_{n}^{\prime}(x, r, t)=\alpha_{n} T+\beta_{n},
$$

where $\boldsymbol{n}$ is the normal vector to the surface $\bar{G}$.

We will consider equation (15) with the initial condition:

$$
T(x, r, 0)=T_{0}(x, r),(x, r) \in \bar{G} .
$$

The next equation is used to calculate the coefficient of conductivity of the water and steam in the international practice:

$$
\lambda=\lambda_{0}(\tau)+\lambda_{1}(\delta)+\lambda_{2}(\tau, \delta)
$$

where $\lambda$ is the conductivity, $\mathrm{W} /(\mathrm{m} \cdot \mathrm{K}) ; \tau=T / T^{*} ; T$ is the absolute temperature, $\mathrm{K} ; T^{*}=647,256 \mathrm{~K} ; \delta=\rho / \rho^{*} ; \rho$ is a density, $\mathrm{kg} / \mathrm{m}^{3} ; \rho^{*}=317,7 \mathrm{~kg} / \mathrm{m}^{3}$. The conductivity of water steam in the state of ideal gas $\lambda_{0}(\tau)$ is determined by the equation:

$$
\begin{gathered}
\lambda_{0}(\tau)=\tau^{0,5} \sum_{\kappa=0}^{3} a_{\kappa} \tau^{\kappa}, a_{0}=0,01028 ; a_{1}=0,02996 ; \\
a_{2}=0,01561 ; a_{3}=-0,004224 .
\end{gathered}
$$

The function $\lambda_{1}(\delta)$ is defined:

$$
\lambda_{1}(\delta)=b_{0}+b_{1} \delta+b_{2} \exp \left\{B_{1}\left(\delta+B_{2}\right)^{2}\right\},
$$

where $b_{0}=-0,39707 ; b_{1}=0,4 ; b_{2}=1,06 ; B_{1}=-0,171$; $B_{2}=2,392$, and the function $\lambda_{2}(\tau, \delta)$ is defined:

$$
\begin{aligned}
& \lambda_{2}(\tau, \delta)=\left(\frac{d_{1}}{10}+d_{2}\right) \delta^{9 / 5} \exp \left[C_{1}\left(1-\delta^{14 / 5}\right)\right]+ \\
& +d_{3} S \delta^{Q} \exp \left[\left(\frac{Q}{1+Q}\right)\left(1-\delta^{1+Q}\right)\right]+d_{4} \exp \left(C_{2} \tau^{3 / 2}+\frac{C_{3}}{\delta^{5}}\right)
\end{aligned}
$$

where $Q$ and $S$ are functions of the magnitude $\Delta \tau=|\tau-1|+C_{4}$ :

$$
Q=2+C_{5} / \Delta \tau^{0,6} ; S= \begin{cases}1 / \Delta \tau & \text { for } \tau \geq 1 ; \\ C_{6} / \Delta \tau^{0,6} & \text { for } \tau<1 .\end{cases}
$$

The coefficients $d_{i}$ and $C_{i}$ have the next values:

$$
\begin{gathered}
d_{1}=0,0701309 ; d_{2}=0,0118520 ; d_{3}=0,00169937 \\
d_{4}=-1,0200 ; C_{1}=0,642857 \\
C_{2}=-4,11717 ; C_{3}=-6,17937 ; C_{4}=0,00308976 ; \\
C_{5}=0,0822994 ; C_{6}=10,0932
\end{gathered}
$$

The equation (14) is applicable with the following values of the temperature and pressure: $p \leq 100 \mathrm{MPa}$ for $0 \leq T \leq 500^{\circ} \mathrm{C} ; \quad p \leq 70 \mathrm{MPa}$ for $500<T \leq 650^{\circ} \mathrm{C}$; $p \leq 40 \mathrm{MPa}$ for $650<T \leq 800^{\circ} \mathrm{C}$.

The error values in the liquid field at the temperatures $25-200{ }^{\circ} \mathrm{C}$ and pressures up to $5 \mathrm{MPa}$ is equaled to $1,5 \%$ at higher temperatures up to $300^{\circ} \mathrm{C}-$ $2 \%$. For the water steam at temperatures up to $550{ }^{\circ} \mathrm{C}$ under pressure $0,1 \mathrm{MPa}$ error is equal to $1,5 \%$, under pressures up to $40 \mathrm{MPa}-3 \%$. The equation (18), in contrast to the theoretical conclusions, determines not infinite, but finite conductivity value at the critical point, which does not allow to estimate the error values near its critical point.

\section{Splitting schemes into physical processes}

The initial aerodynamic model are divided into three subtasks according to the of the pressure amendment method [1-4]. The first subtask is representative by the diffusion-convection problem on the basis of which the velocity field are calculated at the intermediate time step:

$$
\begin{aligned}
& \frac{\tilde{v}_{r}-v_{r}}{\tau}+v_{r} \frac{\partial v_{r}}{\partial r}-\frac{v_{\theta}^{2}}{r}+v_{z} \frac{\partial v_{r}}{\partial z}=\mu\left(\frac{\partial}{\partial r}\left(\frac{1}{r} \frac{\partial\left(r v_{r}\right)}{\partial r}\right)+\frac{\partial^{2} v_{r}}{\partial z^{2}}\right)+f_{r}, \\
& \frac{\hat{v}_{\theta}-v_{\theta}}{\tau}+v_{r} \frac{\partial v_{\theta}}{\partial r}+\frac{v_{\theta} v_{r}}{r}+v_{z} \frac{\partial v_{\theta}}{\partial z}=\mu\left(\frac{\partial}{\partial r}\left(\frac{1}{r} \frac{\partial\left(r v_{\theta}\right)}{\partial r}\right)+\frac{\partial^{2} v_{\theta}}{\partial z^{2}}\right)+f_{\theta}, \\
& \frac{\tilde{v}_{z}-v_{z}}{\tau}+v_{r} \frac{\partial v_{z}}{\partial r}+v_{z} \frac{\partial v_{z}}{\partial z}=\mu\left(\frac{1}{r} \frac{\partial}{\partial r}\left(r \frac{\partial v_{z}}{\partial r}\right)+\frac{\partial^{2} v_{z}}{\partial z^{2}}\right)+f_{z} .
\end{aligned}
$$


Calculation of pressure distribution (the second subtask):

$$
\frac{\partial \rho}{\partial t}+\frac{1}{r} \frac{\partial\left(\rho r \tilde{v}_{r}\right)}{\partial r}+\frac{\partial\left(\rho \tilde{v}_{z}\right)}{\partial z}=\frac{\tau}{r} \frac{\partial}{\partial r}\left(r \frac{\partial P}{\partial r}\right)+\tau \frac{\partial^{2} P}{\partial z^{2}}
$$

or with consideration the state equation

$$
\begin{aligned}
& r \frac{\partial(P M / \varepsilon R T)}{\partial t}+\frac{\partial\left(\rho r \tilde{v}_{r}\right)}{\partial r}+r \frac{\partial\left(\rho \tilde{v}_{z}\right)}{\partial z}= \\
& =\tau \frac{\partial}{\partial r}\left(r \frac{\partial P}{\partial r}\right)+\tau r \frac{\partial^{2} P}{\partial z^{2}} .
\end{aligned}
$$

The simplified hydrostatic model of water motion is used as the initial approximation for this problem. It is significantly decreased the calculation time.

The third subtask is determined the distribution of the steam velocity at the upper time layer by the explicit formulas:

$$
\frac{\hat{v}_{r}-\tilde{v}_{r}}{\tau}=-\frac{1}{\rho} \frac{\partial P}{\partial r}, \frac{\hat{v}_{z}-\tilde{v}_{z}}{\tau}=-\frac{1}{\rho} \frac{\partial P}{\partial z} .
$$

where $\tau$ is a time step; $v$ is a value of velocity field on the previous time layer; $\tilde{v}$ is a value of velocity field on the intermediate time layer; $\hat{v}$ is on the current time layer.

\section{Software description}

The input model parameters are the next: rotation frequency of the turbine, the range of output speeds at the turbine blades, number of blades, the number of nozzle channels, the width of the nozzle channel to the impeller, the vapor pressure of the working wheels. The calculations were performed in three functional areas $1-3$. The location of areas which were determined by the velocity field is given in Fig. 1 .
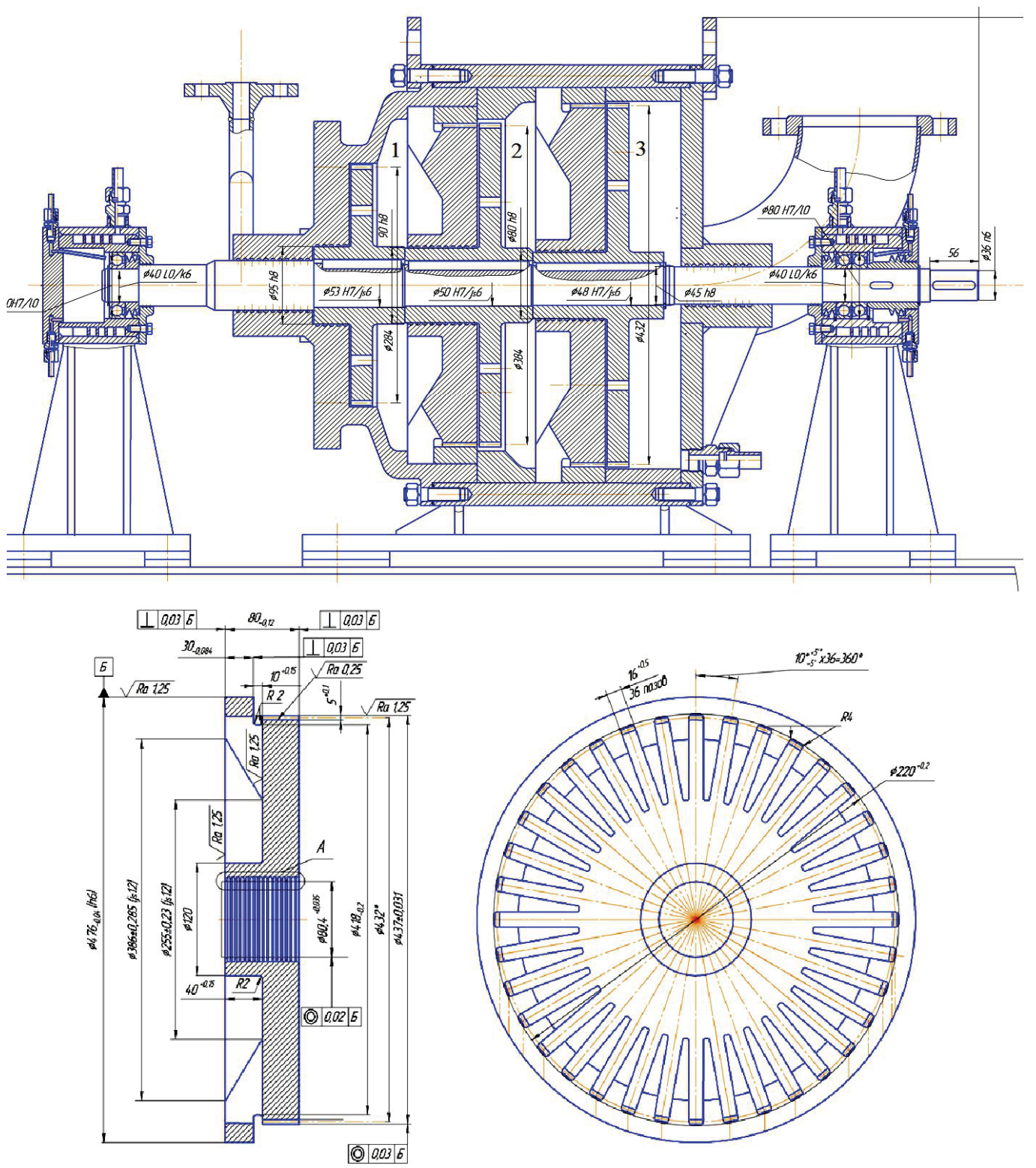

Fig. 1. The geometry of turbine housing and the nozzle grid. 


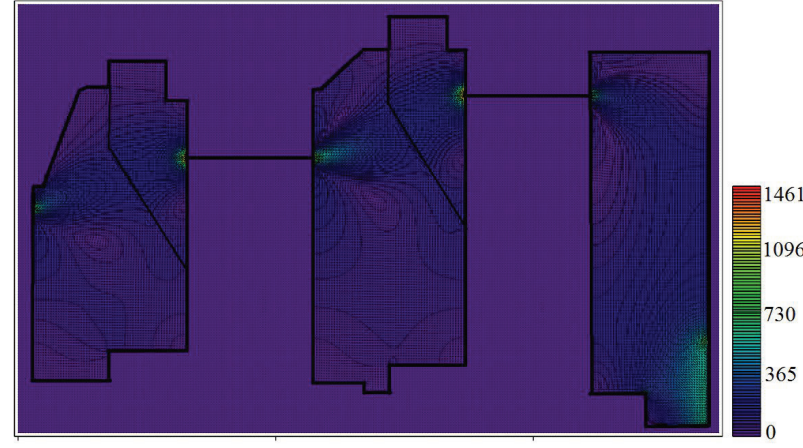

Fig. 2. The velocity of steam movement in working chamber.

The flow pattern inside a pair of working chambers (shown in a longitudinal and radial component of the velocity vector) is given in Fig. 2 .
Color reflects the speed of movement of the steam. The units of measurement are meters per second. The developed software allows to calculate the movement of air environment within areas with cylindrical symmetry and allows to identify patterns of velocity fields and pressure within axial steam turbines. The permeability of the medium is used in the mathematical model to describe the nozzle geometry of the lattice. The developed software can calculate the steam movement in multiple stages at the same time.

Distribution fields of the main physical values calculated for inside the first working chamber ( 1 is the longitudinal and radial component of the velocity vector, $\mathrm{m} / \mathrm{s} ; 2$ is the rotation speed, rev/min; 3 is the pressure, atm.; 4 is the turbulent viscosity, $\mathrm{Pa} \cdot \mathrm{s} ; 5$ is the temperature, ${ }^{\circ} \mathrm{C}$; 6 is the density, $\mathrm{kg} / \mathrm{m}^{3}$ ) are given in Fig. 3 .
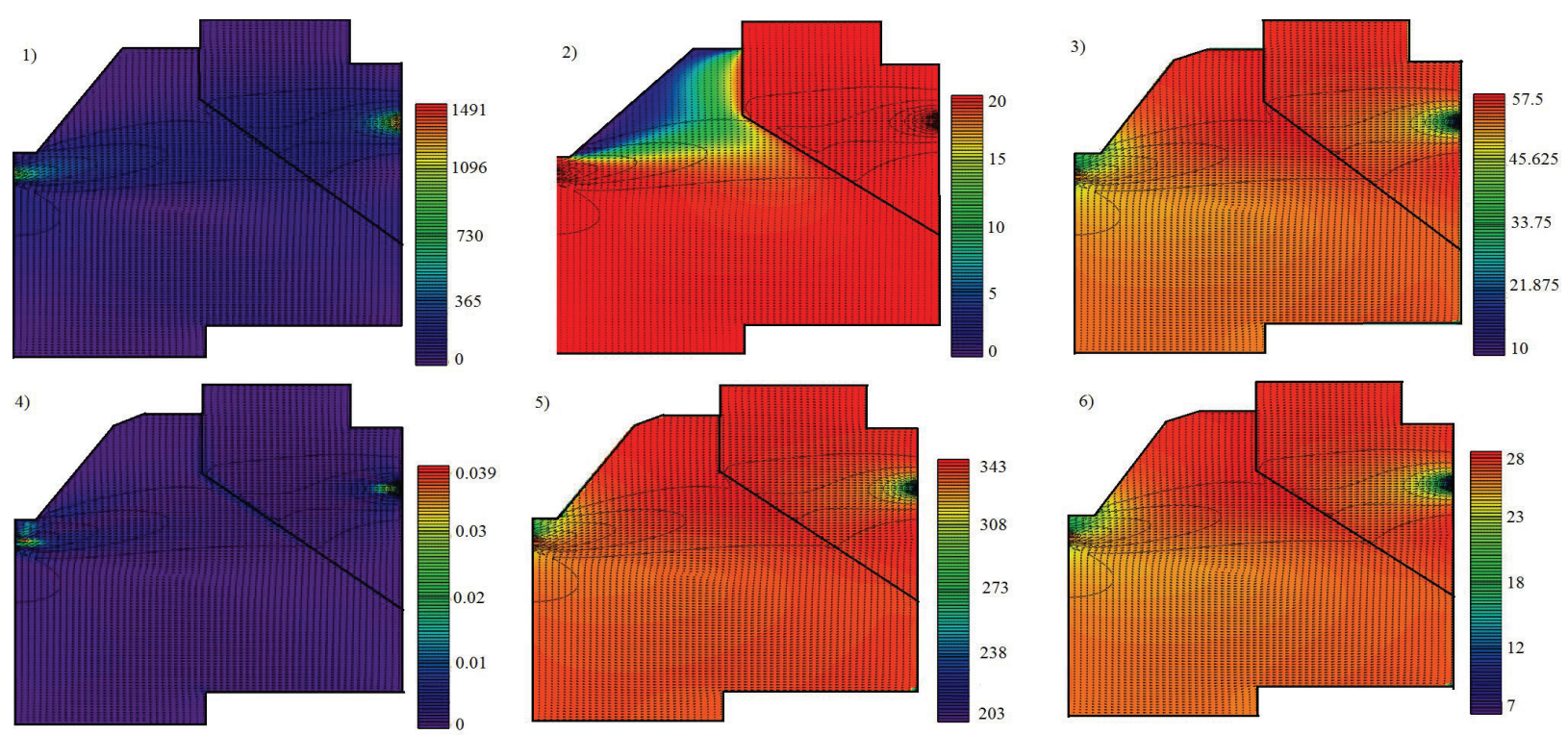

Fig. 3. Distribution fields of the main calculated physical quantities inside for the first working chamber

\section{Conclusion}

The mathematical model of aerodynamic processes in the steam turbine was developed. The main equations are the next: the Navier-Stokes equations, continuity equations and state equations. The initial system of equations was determined in cylindrical coordinate system taking into account the axial symmetry of steam flow field in the turbine. The splitting schemes into physical processes (the pressure amendment method) was used for approximation of problem at the temporary variable. According to this method, the solution problem is calculated in three steps. The solution of diffusionconvection problem with high Peclet numbers is required in calculation of velocity field components without pressure. New difference schemes were obtained on the basis of the modification of the "cabaret" scheme and used for this problem solution. Owning to these difference schemes, the accuracy of diffusion-convection problem solution is increased in 2,5-3 times, compared to the traditional "cabaret" schemes, which are effective for solution such problems. The software implementation was performed on the basis of the developed algorithms. Results of numerical calculations of aerodynamics processes in the steam turbine were obtained.

This work was financially supported by the FTPRD «Research and Development for the Development of the Scientific and Technological Complex of Russia for 2014-2020», Project No. 14.577.21.0260.

\section{References}

1. A.A. Samarsky, E.S. Nikolaev, Methods of solving grid equations (M.: Science, 1978)

2. A.I. Sukhinov, A.E. Chistyakov, J. Mathematical modeling, 24, no. 1, 3-20 (2012)

3. A.N. Konovalov, J. Differential equations, 40, no. 7, (2004)

\footnotetext{
*Corresponding author: cheese_05@mail.ru
} 
4. A.N. Konovalov. Siberian mathematical journal, 43, no. 3, 552 (2002)

5. A.I. Sukhinov, A.E. Chistyakov, A.V. Shishenya J. Mathematical modeling, 25, no. 11, 53-64 (2013)

6. A.I. Sukhinov, A.E. Chistyakov, N.A. Fomenko, J. Izvestiya SFedU engineering sciences, no. 4, 87-98 (2013)

7. A.A. Samarskiy, Theory of difference schemes. (Nauka, Moscow, 1989)

8. A.A. Samarskiy, A.V. Gulin, Numerical methods (Nauka, Moscow, 1989)

9. K.A. Beklemishev, I.B. Petrov, A.V. Favorsky, J. Mathematical modeling, 25, no. 11, 3-16 (2013)

10. I.B. Petrov, A.V. Favorsky, A.V. Sannikov, I.E. Kvasov, J. Mathematical modeling, 25, no. 2, $42-52$ (2013)

11. A.I. Sukhinov, A.E. Chistyakov, A.A. Semenyakina A.V. Nikitina, J. Computational methods and programming: new computing technology, 16, no. 2 , 256-267 (2015)

12. A.V. Nikitina, A.A. Semenyakina, A.E.Chistyakov, E.A. Protsenko, I.V. Yakovenko, J. Fundamental research, no. 12-3, 500-504 (2015)

13. A.E. Chistyakov, D.S. Hachunts, A.V. Nikitina, E.A. Protsenko, I.Yu. Kuznetsova, J. Modern problems of science and education, no. 1-1, 1786 (2015)

14. A.I. Sukhinov, A.V. Nikitina, A.A. Semenyakina, E.A. Protsenko, J. Engineering journal of Don, 38, no. 4 (38), 52 (2015)

15. A.V. Nikitina, Y.A. Abramenko, A.E. Chistyakov, J. Informatics, computer science and engineering education, no. 3 (23), 49-55 (2015)

16. A.E. Chistyakov, A.V. Nikitina, G.A. Ougolnitsky V.M. Puchkin, I.S. Semenov, A.I. Sukhinov, A.B. Usov, J. Game Theory and Applications, 17, 37-48 (2015)

17. A.E. Chistyakov, A.V.Nikitina, V.V. Sumbaev, J. Herald of computer and information technologies, no. 8 (146), 3-7 (2016)

18. A.V. Nikitina, A.A. Semenyakina, A.E. Chistyakov, J. Herald of computer and information technologies, no. 7 (145), 3-8 (2016)

19. A.I. Sukhinov, A.E. Chistyakov, A.A. Semenyakina A.V. Nikitina, J. Computer researches and modeling, 8, no. 1, $151-168$ (2016)

20. A.I. Sukhinov, A.V. Nikitina, A.A. Semenyakina, A.E. Chistyakov, CEUR Workshop Proceedings, 10th Annual International Scientific Conference on Parallel Computing Technologies, PCT 2016; 1576, 308-319, (2016)

21. A.V. Nikitina, A.I. Sukhinov, G.A. Ugolnitsky, A.B. Usov, A.E. Chistyakov, M.V. Puchkin, I.S. Mathematical Models and Computer Simulations. 2017. 9 (1), 101-107.

22. A.I. Sukhinov, A.E. Chistyakov, I.I. Levin, I.S. Semenov, A.V. Nikitina, A.A. Semenyakina 5th
International Conference on Informatics, Electronics and Vision (ICIEV), 1128 - 1133 (2016)

23. A.I. Sukhinov, A.E. Chistyakov, A.V. Nikitina A.A. Semenyakina, I. Korovin, G. Schaefer Conference on Informatics, Electronics and Vision (ICIEV). 1134 - 1139, (2016)

24. A.I. Sukhinov, A.E. Chistyakov, A.A. Semenyakina, A.V. Nikitina Computer researches and modeling. 8, no. 1., $151-168$ (2016)

25. A.I. Sukhinov, D.S. Khachunts, A.E. Chistyakov, Computational Mathematics and Mathematical Physics, 55, No. 7., 1216-1231 (2015)

26. A.I. Sukhinov, A.E. Chistyakov, A.V. Shishenya Mathematical Models and Computer Simulations. 6, No. 3.,324-331 (2014)

27. N. Buzalo, P. Ermachenko, T. Bock, A. Bulgakov A.Chistyakov, A. Sukhinov, E. Zhmenya, N. Zakharchenko Procedia Engineering, 85, No. C., 84-93 (2014)

28. A.I. Sukhinov, A.E. Chistyakov, E.F. Timofeeva, A.V. Shishenya, Mathematical Models and Computer Simulations, 5, No. 2, 122-129, 2013

29. A.I. Sukhinov, A.E. Chistyakov, Mathematical Models and Computer Simulations, 4, Iss. 4, 398409, (2012) 